\title{
EDITORIAL
}

\section{Explainable Artificial Intelligence: A New Era of Artificial Intelligence}

\author{
Ashraf Darwish \\ Faculty of Science, Helwan University, Cairo, Egypt
}

Received: 7 January 2022; Accepted: 12 January 2022; Published: 19 January 2022

Recently, Artificial Intelligence (AI) has emerged as an emerging with advanced methodologies and innovative applications. With the rapid advancement of AI concepts and technologies, there has been a recent trend to add interpretability and explainability to the paradigm. With the increasing complexity of AI applications, their a relationship with data analytics, and the ubiquity of demanding applications in a variety of critical applications such as medicine, defense, justice and autonomous vehicles, there is an increasing need to associate the results with sound explanations to domain experts. All of these elements have contributed to Explainable Artificial Intelligence (XAI) ${ }^{[1,2]}$. Therefore, XAI can explain how AI obtained a particular result. In addition, algorithms can be used efficiently to solve prediction, classification and regression problems. For numerous crucial fields of human existence, blackbox machine learning models are fast being deployed with a tag of AI-enabled technology. Therefore, this explainability and transparency requirements lead a new area of AI research, known as XAI. XAI can enhance fairness and transparency by providing an explanation or justification that can be understood by human. On the other hand, the transparent model is realized with some algorithms such as Linear Regression, Decision Trees and Rule based learning.

Many approaches such as LIME and SHAP, provenance and taxonomy inductions, and counter factual explanations may be used on various types of data such as text, picture, audio, and video ${ }^{[3]}$.

Any form of supervised learning model's prediction may be justified with the help of LIME. This method may be used with any type of data, including images, text, and video. LIME can handle any supervised learning model and offer explanation in this way. LIME generates local optimal explanations by computing essential aspects in the immediate neighborhood of the instance to be explained. In the field of XAI, LIME has found a lot of success and support, and it's used for text, picture, and tabular data. LIME is relevant and expandable to all key machine learning fields, which is a noteworthy feature.

The fundamental goal of SHAP is to compute the decision-making contribution of each feature for classification in order to comprehend the prediction of an input. SHAP uses coalitional game theory to calculate Shapley values. It's a strategy that Shapley describes as a method for providing a reward to game players based on

*Corresponding Author:

Ashraf Darwish,

Faculty of Science, Helwan University, Cairo, Egypt;

Email: ashraf.darwish.eg@ieee.org

DOI: https://doi.org/10.54963/dtra.v1i1.29

Copyright (C) 2022 by the author(s). Published by UK Scientific Publishing Limited. This is an open access article under the Creative Commons Attribution (CC BY) license (https://creativecommons.org/licenses/by/4.0/). 
their participation to the game.

It is highlighted here that XAI is a significant and required feature of real-time Artificial Intelligence applications. The major objectives of XAI is transparency, fairness, bias, and confidence ${ }^{[4]}$. This editorial presents a conceptual understanding of XAI as well as the significance of explainability in motivating academics and researchers to investigate various facets and develop methods and techniques of XAI.

\section{References}

[1] Gohel, et al., 2017. Explainable AI: current status and future directions, Digital Object Identifier 10.1109.

[2] Angelov, P.P., Soares, E.A., Jiang, R., Arnold, N.I.,
Atkinson, P.M., 2021. Explainable artificial intelligence: an analytical review. Wiley Interdisciplinary Reviews: Data Mining and Knowledge Discovery. 11(5), e1424.

DOI: https://doi.org/10.1002/widm.1424.

[3] Soares, E., Angelov, P., Biaso, S., Higa Froes, M., Daniel Kanda Abe. SARS-CoV-2 CT-scan dataset: A large dataset of real patients CT scans for SARSCoV-2 identification.

DOI: https://doi.org/10.1101/2020.04.24.20078584.

[4] Witold Pedrycz Shyi-Ming Chen, Interpretable Artificial Intelligence: A Perspective of Granular Computing. Studies in Computational Intelligence. Volume 937.

DOI: https://doi.org/10.1007/978-3-030-64949-4. 